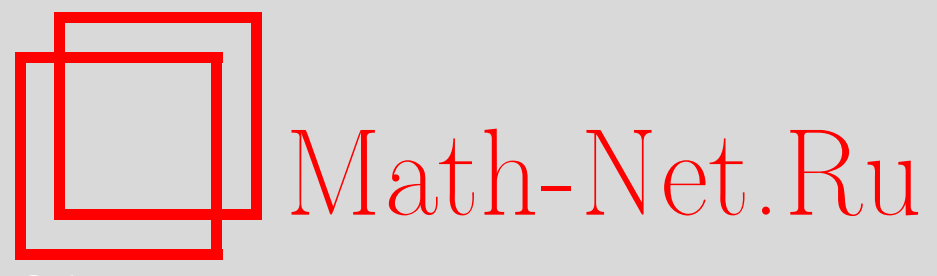

Г. И. Архипов, В. Н. Чубариков, Асимптотическая формула для числа точек решетки в круге на плоскости Лобачевского, Дискрет. матем., 2006, том 18, выпуск 4, 9-17

DOI: https://doi.org/10.4213/dm69

Использование Общероссийского математического портала Math-Net.Ru подразумевает, что вы прочитали и согласны с пользовательским соглашением http://www.mathnet.ru/rus/agreement

Параметры загрузки:

IP : 3.85 .5 .30

26 апреля 2023 г., 17:51:59

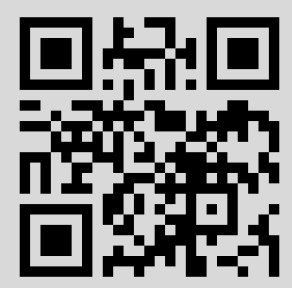




\title{
Асимптотическая формула для числа точек решетки в круге на плоскости Лобачевского
}

\author{
() 2006 г. Г. И. Архипов, В. Н. Чубариков
}

Для точек $z=x+i y$ и $z^{\prime}=x^{\prime}+i y^{\prime}$ из верхней полуплоскости определим расстояние $d=d\left(z, z^{\prime}\right)$, полагая

$$
d=\ln \left(\frac{u+2+\sqrt{u^{2}+4 u}}{2}\right),
$$

где

$$
u=\frac{\left|z-z^{\prime}\right|^{2}}{y y^{\prime}}
$$

Круг $K\left(z_{0}, T\right)$ с центром в точке $z_{0}$ состоит из точек $z$, удовлетворяющих неравенству $d\left(z, z_{0}\right) \leqslant T$. Пусть $N\left(z_{0}, T\right)$ - число элементов $\gamma$ модулярной группы $P S L_{2}(\mathbf{Z})$ таких, что точка $\gamma z_{0}$ попадает в круг $K\left(z_{0}, T\right)$. В работе уточняется остаточный член в асимптотической формуле для величины $N\left(z_{0}, T\right)$.

Пусть $z=x+i y-$ комплексное число, лежащее в верхней полуплоскости комплексной плоскости. В модели Клейна верхняя полуплоскость определяет плоскость Лобачевского с помощью метрики $l=l(z)$, задаваемой равенством

$$
d l^{2}=\frac{d x^{2}+d y^{2}}{y^{2}} .
$$

Группа $G=S L_{2}(\mathbf{R})$ вещественных матриц второго порядка

$$
\gamma=\left(\begin{array}{ll}
a & b \\
c & d
\end{array}\right)
$$

с определителем, равным 1, с помощью дробно-линейных преобразований

$$
\gamma z=\frac{a z+b}{c z+d}
$$

задает группу движений на плоскости Лобачевского. Отметим, что эта метрика является $G$-инвариантной, то есть

$$
d l^{2}(z)=d l^{2}(\gamma z)
$$


Метрика $l$ определяет геодезическое расстояние $d=d\left(z, z^{\prime}\right)$ между точками $z=x+i y$ и $z^{\prime}=x^{\prime}+i y^{\prime}$ из верхней полуплоскости следующим образом. Положим

$$
u=u\left(z, z^{\prime}\right)=\frac{\left|z-z^{\prime}\right|^{2}}{y y^{\prime}} .
$$

Тогда расстояние $d=d\left(z, z^{\prime}\right)$ задается равенством

$$
d=\ln \left(\frac{u+2+\sqrt{u^{2}+4 u}}{2}\right)
$$

Функция расстояния $d$ является $G$-инвариантной так же, как и метрика $l$, то есть

$$
d\left(z, z^{\prime}\right)=d\left(\gamma z, \gamma z^{\prime}\right)
$$

Круг $K\left(z_{0}, T\right)$ с центром в точке $z_{0}$ и радиусом $T$ состоит из точек $z$, удовлетворяющих неравенству $d\left(z, z_{0}\right) \leqslant T$. Последнее неравенство можно переписать в виде

$$
\left(x-x_{0}\right)^{2}+\left(y-y_{0} \operatorname{ch} T\right)^{2} \leqslant y_{0}^{2} \operatorname{sh}^{2} T .
$$

Обозначим $N\left(z_{0}, T\right)$ число элементов $\gamma$ модулярной группы $P S L_{2}(\mathbf{Z})=S L_{2}(\mathbf{Z}) / \pm I$ таких, что точка $\gamma z_{0}$ попадает в круг $K\left(z_{0}, T\right)$.

В настоящей работе уточняется остаточный член в асимптотической формуле для величины $N\left(z_{0}, T\right)$. В случае, когда $z_{0}=i$, задача получения асимптотической формулы для $N(T)=N(i, T)$ является прямым аналогом проблемы Гаусса о числе целых точек в круге $x^{2}+y^{2} \leqslant T$ на евклидовой плоскости.

Сначала сформулируем утверждение (см. лемму 1 на с. 301 в [17]), которое связывает величину $N(T)$ с числом целых точек на некоторой поверхности.

Лемма 1. Величина $2 N(T)$ равна числу цельх точек на поверхности ad $-b c=1 c$ условием $a^{2}+b^{2}+c^{2}+d^{2} \leqslant 2 \operatorname{ch} T$.

Докажем теперь следующую теорему.

Теорема 1. При $T \rightarrow \infty$

$$
N(T)=8 \operatorname{ch} T+O\left(e^{3 T / 4} T^{4}\right) .
$$

Доказательство. Введем обозначение $R=\operatorname{ch} T$. Из леммы 1 следует, что

$$
2 N(T)=\sum_{\substack{a d-b c=1 \\ a^{2}+b^{2}+c^{2}+d^{2} \leqslant 2 R}} 1=2 \Sigma_{1}-\Sigma_{2},
$$

где

$$
\Sigma_{1}=\sum_{\begin{array}{c}
a d-b c=1 \\
a^{2}+b^{2}+c^{2}+d^{2} \leqslant 2 R \\
a^{2}+b^{2} \leqslant R
\end{array}} 1, \quad \Sigma_{2}=\sum_{\begin{array}{c}
a d-b c=1 \\
a^{2}+b^{2} \leqslant R \\
c^{2}+d^{2} \leqslant R
\end{array}} 1 .
$$

Поскольку $(a, b)=1$, справедливо либо $|a| \neq|b|$, либо $|a|=|b|=1$.

В случае, если $|a|=|b|=1$, число решений неравенства $a^{2}+b^{2}+c^{2}+d^{2} \leqslant 2 R$ при условии $a d-b c=1$ является величиной порядка $\ll \sqrt{R}$.

Если $|a| \neq|b|$, то имеется две возможности: либо $|a|>|b| \geqslant 1$, либо $|b|>|a| \geqslant 1$. 
Преобразуем суммы $\Sigma_{1}$ и $\Sigma_{2}$. Пусть сначала имеет место первая возможность. При фиксированных $a$ и $b$ решим уравнение $a d-b c=1$. Пусть вычет $\beta$ по модулю $a-$ решение сравнения $-\beta b \equiv 1(\bmod a)$. Тогда $c \equiv \beta(\bmod a)$, то есть для любого целого числа $t$ число $c$ можно представить в виде $c=\beta+a t$. Из равенства $a d-b(\beta+a t)=1$ находим, что

$$
d=\frac{\beta b+1}{a}+b t=\alpha+b t
$$

где $\alpha=(\beta b+1) / a$.

Отсюда следует, что суммирование по наборам $(a, b, c, d)$ в $\Sigma_{1}$ можно заменить на суммирование по наборам $(a, b, t)$ с $a^{2}+b^{2} \leqslant R$ и

$$
a^{2}+b^{2}+(\beta+a t)^{2}+(\alpha+b t)^{2} \leqslant 2 R .
$$

Последнее условие эквивалентно неравенству

$$
\left(a^{2}+b^{2}\right) t^{2}+2(a \beta+b \alpha) t+\left(a^{2}+b^{2}+\alpha^{2}+\beta^{2}-2 R\right) \leqslant 0 .
$$

Его решением при фиксированных $a$ и $b$ являются целые точки $t$ отрезка $\left[t_{2}, t_{1}\right]$, где

$$
t_{1,2}=\frac{-(a \beta+b \alpha) \pm \sqrt{\left(2 R-a^{2}-b^{2}\right)\left(a^{2}+b^{2}\right)-1}}{a^{2}+b^{2}} .
$$

В сумме $\Sigma_{2}$ аналогично суммирование по наборам $(a, b, c, d)$ заменяется на суммирование по наборам $(a, b, t)$, где $a^{2}+b^{2} \leqslant R$ и

$$
(a t+\beta)^{2}+(b t+\alpha)^{2} \leqslant R .
$$

Решением последнего неравенства при фиксированных взаимно простых числах $a$ и $b$ являются целые числа $t$ из отрезка $\left[t_{4}, t_{3}\right]$, где

$$
t_{3,4}=\frac{-(a \beta+b \alpha) \pm \sqrt{R\left(a^{2}+b^{2}\right)-1}}{a^{2}+b^{2}} .
$$

Преобразуем теперь суммы $\Sigma_{1}$ и $\Sigma_{2}$ во втором случае, когда $|b|>|a| \geqslant 1$. При фиксированных $a$ и $b$ решения уравнения $a d-b c=1$ задаются в виде $c=\beta+a s$, $d=\alpha+b s$, где числа $\alpha$ и $\beta$ определяются из условий $a \alpha \equiv 1(\bmod b), \beta=(a \alpha-1) / b$.

Итак, во втором случае суммирование в $\Sigma_{1}$ ведется по наборам $(a, b, s)$, где $a$ и $b-$ взаимно простые числа такие, что $a^{2}+b^{2} \leqslant R$, а при фиксированных $a$ и $b$ переменная суммирования $s$ пробегает все целые значения из отрезка $\left[s_{2}, s_{1}\right]$, где

$$
s_{1,2}=\frac{-(a \beta+b \alpha) \pm \sqrt{\left(2 R-a^{2}-b^{2}\right)\left(a^{2}+b^{2}\right)-1}}{a^{2}+b^{2}} .
$$

Аналогично, во втором случае в сумме $\Sigma_{2}$ суммирование ведется по наборам $(a, b, s)$, где $a$ и $b$ - взаимно простые числа такие, что $a^{2}+b^{2} \leqslant R$, а при фиксированных $a$ и $b$ переменная суммирования $s$ пробегает все целые значения из отрезка $\left[s_{4}, s_{3}\right]$, где

$$
s_{3,4}=\frac{-(a \beta+b \alpha) \pm \sqrt{R\left(a^{2}+b^{2}\right)-1}}{a^{2}+b^{2}} .
$$


Таким образом, в силу того, что переменные $s$ и $t$ пробегают одно и то же множество значений,

$$
\Sigma_{1}=2 \sum_{\substack{a^{2}+b^{2} \leqslant R \\|a|>|b|}}^{\prime}\left(\left[t_{2}\right]+\left[-t_{1}\right]+1\right), \quad \Sigma_{2}=2 \sum_{\substack{a^{2}+b^{2} \leqslant R \\|a|>|b|}}^{\prime}\left(\left[t_{4}\right]+\left[-t_{3}\right]+1\right),
$$

где знак штрих в этих суммах означает, что суммирование ведется по взаимно простым числам $a$ и $b$.

Отсюда находим, что

$$
\begin{aligned}
& \Sigma_{1}=2 \sum_{\substack{a^{2}+b^{2} \leqslant R \\
|a|>|b|}}^{\prime}\left(t_{2}-t_{1}+\rho\left(t_{2}\right)+\rho\left(-t_{1}\right)\right), \\
& \Sigma_{2}=2 \sum_{\substack{a^{2}+b^{2} \leqslant R \\
|a|>|b|}}^{\prime}\left(t_{4}-t_{3}+\rho\left(t_{4}\right)+\rho\left(-t_{3}\right)\right),
\end{aligned}
$$

где $\rho(t)=1 / 2-\{t\}$, причем символ $\{t\}$ обозначает дробную часть числа $t$.

Следовательно,

$$
N(T)=\Sigma_{1}-(1 / 2) \Sigma_{2}=S_{0}+2 S_{1}+2 S_{2}-S_{3}-S_{4},
$$

где

$$
S_{0}=\sum_{\substack{a^{2}+b^{2} \leqslant R \\|a|>|b|}}^{\prime} f(a, b), \quad S_{m}=\sum_{\substack{a^{2}+b^{2} \leqslant R \\|a|>|b|}}^{\prime} \rho\left((-1)^{m} t_{m}\right), \quad m=1,2,3,4,
$$

причем

$$
f(a, b)=\frac{4 \sqrt{\left(2 R-a^{2}-b^{2}\right)\left(a^{2}+b^{2}\right)-1}-2 \sqrt{R\left(a^{2}+b^{2}\right)-1}}{a^{2}+b^{2}} .
$$

Найдем асимптотическую формулу для суммы $S_{0}$. Справедливы равенства

$$
S_{0}=\sum_{\substack{a^{2}+b^{2} \leqslant R \\|a|>|b|}}^{\prime} f(a, b)=\sum_{\substack{a^{2}+b^{2} \leqslant R \\|a|>|b| \geqslant 1}} f(a, b) \sum_{r \mid(|a|,|b|)} \mu(r)=\sum_{r \leqslant R^{1 / 2}} \mu(r) W,
$$

где

$$
W=W(r)=\sum_{\substack{a^{2}+b^{2} \leqslant R r^{-2} \\|a|>|b| \geqslant 1}} f(a r, b r)=4 \sum_{\substack{a^{2}+b^{2} \leqslant R r^{-2} \\ a>b \geqslant 1}} f(a r, b r) .
$$

Преобразуем двойную сумму $W$ к повторной сумме. Получим, что

$$
W=4 \sum_{1 / 2<a \leqslant R^{1 / 2} r^{-1}} V, \quad V=V(a)=\sum_{1 / 2<b \leqslant\left(R r^{-2}-a^{2}\right)^{1 / 2}} f(a r, b r) .
$$

К внутренней сумме $V$ применим формулу Эйлера суммирования значений функции по переменной $b$ (см., например, теорему 4 на с. 206 в [3]). Для $V$ справедливо представление

$$
V=V_{1}+V_{2}-V_{3} \text {, }
$$


где

$$
\begin{aligned}
& V_{1}=\int_{1 / 2}^{\left(R r^{-2}-a^{2}\right)^{1 / 2}} f(a r, y r) d y, \\
& V_{2}=\rho\left(\left(R r^{-2}-a^{2}\right)^{1 / 2}\right) f\left(a r,\left(R-a^{2} r^{2}\right)^{1 / 2}\right), \\
& V_{3}=-\int_{1 / 2}^{\left(R r^{-2}-a^{2}\right)^{1 / 2}} \rho(y) f_{y}^{\prime}(a r, y r) d y .
\end{aligned}
$$

Просуммируем $V_{1}=V_{1}(a)$ по промежутку, определяющему внешнее суммирование по переменной $a$ в сумме $W$, а затем к получившейся сумме применим формулу суммирования Эйлера. Получим, что

$$
\begin{aligned}
W_{1} & =4 \sum_{1 / 2<a \leqslant R^{1 / 2} r^{-1}} V_{1}(a)=4 \sum_{1 / 2<a \leqslant R^{1 / 2} r^{-1}} \int_{1 / 2}^{\left(R r^{-2}-a^{2}\right)^{1 / 2}} f(a r, y r) d y \\
& =4 \int_{1 / 2}^{R^{1 / 2} r^{-1}}\left(\sum_{1 / 2<a \leqslant\left(R r^{-2}-y^{2}\right)^{1 / 2}} f(a r, y r)\right) d y \\
& =W_{11}+W_{12}+W_{13},
\end{aligned}
$$

где

$$
\begin{aligned}
& W_{11}=4 \int_{1 / 2}^{R^{1 / 2} r^{-1}} d y \int_{1 / 2}^{\left(R r^{-2}-y^{2}\right)^{1 / 2}} f(x r, y r) d x, \\
& W_{12}=4 \int_{1 / 2}^{R^{1 / 2} r^{-1}} \rho\left(\left(R r^{-2}-y^{2}\right)^{1 / 2}\right) f\left(\left(R-y^{2} r^{2}\right)^{1 / 2}, y r\right) d y, \\
& W_{13}=-4 \int_{1 / 2}^{R^{1 / 2} r^{-1}} d y \int_{1 / 2}^{\left(R r^{-2}-y^{2}\right)^{1 / 2}} \rho(x) f_{x}^{\prime}(x r, y r) d x .
\end{aligned}
$$

Вычисляя сначала интеграл $W_{11}$, находим, что

$$
\begin{aligned}
W_{11} & =4 \int_{0}^{\pi / 2} d \varphi \int_{1 / 2}^{\sqrt{R} r^{-1}} \frac{4 \sqrt{\left(2 R-\rho^{2} r^{2}\right) \rho^{2} r^{2}-1}-2 \sqrt{R \rho^{2} r^{2}-1}}{\rho r^{2}} d \rho+O\left(\sqrt{R} r^{-2}\right) \\
& =\frac{2 \pi R}{r^{2}} \int_{r /(2 \sqrt{R})}^{1} \frac{4 \sqrt{\left(2-\rho^{2}\right) \rho^{2}-1 / R^{2}}-2 \sqrt{\rho^{2}-1 / R^{2}}}{\rho} d \rho+O\left(\sqrt{R} r^{-2}\right) \\
& =\frac{2 \pi R}{r^{2}} \int_{0}^{1}\left(4 \sqrt{2-\rho^{2}}-2\right) d \rho+O\left(\sqrt{R} r^{-2}\right) \\
& =\left.\frac{2 \pi R}{r^{2}}\left(4 \arcsin \frac{\rho}{2}+4 \frac{\rho \sqrt{2-\rho^{2}}}{2}-2 \rho\right)\right|_{0} ^{1}+O\left(\sqrt{R} r^{-2}\right) \\
& =\frac{4 \pi^{2} R}{3 r^{2}}+O\left(\sqrt{R} r^{-2}\right) .
\end{aligned}
$$

Оценим сверху интеграл $W_{12}$. Поскольку $f\left(\left(R-y^{2} r^{2}\right)^{1 / 2}, y r\right) \ll 1$, справедлива оценка

$$
W_{12}=4 \int_{1 / 2}^{R^{1 / 2} r^{-1}} \rho\left(\left(R r^{-2}-y^{2}\right)^{1 / 2}\right) f\left(\left(R-y^{2} r^{2}\right)^{1 / 2}, y r\right) d y \ll \sqrt{R} r^{-1} .
$$


Наконец, оценим интеграл $W_{13}$. При $x^{2}+y^{2} \leqslant R r^{-2}$ справедливо неравенство $f_{x}^{\prime}(x r, y r) \leqslant 0$, поэтому

$$
\begin{aligned}
\left|W_{13}\right| & =4\left|\int_{1 / 2}^{R^{1 / 2} r^{-1}} d y \int_{1 / 2}^{\left(R r^{-2}-y^{2}\right)^{1 / 2}} \rho(x) f_{x}^{\prime}(x r, y r) d x\right| \\
& \leqslant 4 \int_{1 / 2}^{R^{1 / 2} r^{-1}} d y \int_{1 / 2}^{\left(R r^{-2}-y^{2}\right)^{1 / 2}}\left|f_{x}^{\prime}(x r, y r)\right| d x \\
& =4 \int_{1 / 2}^{R^{1 / 2} r^{-1}} d y \int_{1 / 2}^{\left(R r^{-2}-y^{2}\right)^{1 / 2}}\left(-f_{x}^{\prime}(x r, y r)\right) d x \\
& \leqslant 4 \int_{1 / 2}^{R^{1 / 2} r^{-1}} f(r / 2, y r) d y \ll \sqrt{R} r^{-1} \int_{1 / 2}^{R^{1 / 2} r^{-1}} \frac{d y}{y} \ll \sqrt{R} r^{-1} \ln R .
\end{aligned}
$$

Таким образом, получаем, что

$$
W_{1}=\frac{4 \pi^{2} R}{3 r^{2}}+O\left(\sqrt{R} r^{-1} \ln R\right)
$$

Оценим сумму $W_{2}$. Поскольку $f\left(a r,\left(R-a^{2} r^{2}\right)^{1 / 2}\right) \ll 1$, справедлива оценка

$$
W_{2}=4 \sum_{1 / 2<a \leqslant R^{1 / 2} r^{-1}} \rho\left(\left(R r^{-2}-a^{2}\right)^{1 / 2}\right) f\left(a r,\left(R-a^{2} r^{2}\right)^{1 / 2}\right) \ll \sqrt{R} r^{-1} .
$$

Далее оценим $W_{3}$. При $a^{2}+y^{2} \leqslant R r^{-2}$ справедливо неравенство $f_{y}^{\prime}(a r, y r) \leqslant 0$, поэтому

$$
\begin{aligned}
\left|W_{3}\right| & =4\left|\sum_{1 / 2 \leqslant a \leqslant R^{1 / 2} r^{-1}} \int_{1 / 2}^{\left(R r^{-2}-a^{2}\right)^{1 / 2}} \rho(y) f_{y}^{\prime}(a r, y r) d y\right| \\
& \leqslant 4 \sum_{1 / 2 \leqslant a \leqslant R^{1 / 2} r^{-1}} \int_{1 / 2}^{\left(R r^{-2}-a^{2}\right)^{1 / 2}}\left|f_{y}^{\prime}(a r, y r)\right| d y \\
& =4 \sum_{1 / 2 \leqslant a \leqslant R^{1 / 2} r^{-1}} \int_{1 / 2}^{\left(R r^{-2}-a^{2}\right)^{1 / 2}}\left(-f_{y}^{\prime}(a r, y r)\right) d y \\
& \leqslant 4 \sum_{1 / 2 \leqslant a \leqslant R^{1 / 2} r^{-1}} f(a r, r / 2) \ll \sqrt{R} r^{-1} \sum_{1 / 2 \leqslant a \leqslant R^{1 / 2} r^{-1}} \frac{1}{a} \ll \sqrt{R} r^{-1} \ln R .
\end{aligned}
$$

Наконец, собирая вместе все найденные результаты, получаем асимптотическую формулу

$$
S_{0}=\frac{4 \pi^{2} R}{3 r^{2}}+O\left(\sqrt{R} r^{-1} \ln R\right)
$$

Осталось оценить сверху модули сумм $S_{1}, S_{2}, S_{3}, S_{4}$. С этой целью воспользуемся разложением в ряд Фурье функции $\rho(t)$ с остатком, а затем разложением остатка в ряд Фурье (см., например, теорему 1 на с. 440 и лемму 1 на с. 601 в [3]). При некотором $N \geqslant 2$

$$
\rho(t)=\sum_{n=1}^{N} \frac{\sin 2 \pi n t}{\pi n}+O\left(\psi_{N}(t)\right)
$$


где

$$
\psi_{N}(t)=\sum_{m=-\infty}^{\infty} c_{m} e^{2 \pi i m t}, \quad\left|c_{m}\right| \leqslant \frac{4+\ln N}{N} e^{-|m| / N} .
$$

Следователฺьно,

$$
\begin{aligned}
S_{m}=\sum_{\substack{a^{2}+b^{2} \leqslant R \\
|a|>|b|}}^{\prime} \rho\left(t_{m}\right) & \ll \sum_{n=1}^{N} \frac{1}{n}\left|\sum_{\substack{a^{2}+b^{2} \leqslant R \\
|a|>|b|}}^{\prime} e^{2 \pi i n t_{m}}\right| \\
& +\frac{\ln N}{N} \sum_{n=1}^{N} \sum_{\substack{a^{2}+b^{2} \leqslant R \\
|a|>|b|}}^{\prime} 1+\frac{\ln N}{N} \sum_{k=1}^{[N \ln N]}\left|\sum_{\substack{a^{2}+b^{2} \leqslant R \\
|a|>|b|}}^{\prime} e^{2 \pi i k t_{m}}\right|+\frac{R \ln N}{N} .
\end{aligned}
$$

Положим $N=\left[R^{1 / 4}\right]$. Далее при $1 \leqslant n \leqslant[N \ln N]$ достаточно оценить модуль суммы

$$
U_{n}=\sum_{\substack{a^{2}+b^{2} \leqslant R \\ a>b \geqslant 1}}^{\prime} e^{2 \pi i n t_{m}}
$$

Рассмотрим сначала

$$
\begin{aligned}
t_{1,2} & =\frac{-(a \beta+b \alpha) \pm \sqrt{\left(2 R-a^{2}-b^{2}\right)\left(a^{2}+b^{2}\right)-1}}{a^{2}+b^{2}} \\
& =-\frac{\beta}{a}-\frac{b}{a\left(a^{2}+b^{2}\right)} \pm \frac{\sqrt{\left(2 R-a^{2}-b^{2}\right)\left(a^{2}+b^{2}\right)-1}}{a^{2}+b^{2}} \\
& =-\frac{\beta}{a}+g(a, b), \quad-\beta b \equiv 1 \quad(\bmod a) .
\end{aligned}
$$

Преобразуем сумму $U_{n}$. Положим $b \bar{b} \equiv 1(\bmod a)$. Воспользуемся разложением в ряд Фурье функции

$$
K(b)=K_{n}(b, a)= \begin{cases}e^{-2 \pi i n \bar{b} / a}, & \text { если }(b, a)=1 \\ 0, & \text { если }(b, a)>1\end{cases}
$$

В разложении

$$
K(b)=\sum_{k=1}^{a} f_{k} e^{2 \pi i n b k / a}
$$

коэффициенты Фурье $f_{s}$ равны

$$
f_{s}=\frac{1}{a} \sum_{m=1}^{a} K(m) e^{-2 \pi i m s / a}=\frac{1}{a} \sum_{m=1,(m, a)=1}^{a} e^{-2 \pi i(n \bar{m}+s m / a}, \quad s=1,2, \ldots, a .
$$

Эти коэффициенты Фурье представляют собой суммы Клостермана по модулю $а$. Из оценок А. Вейля и Салье (см., например, [18], с. 44) следует, что

$$
\left|f_{s}\right| \leqslant \tau(a) a^{-1 / 2}(n, a)^{1 / 2},
$$


где функщия $\tau(a)$ обозначает количество делителей числа $a$.

Далее,

$$
\begin{aligned}
U_{n} & =\sum_{a^{2}+b^{2} \leqslant R} e^{2 \pi i n g(a, b)} K_{n}(b, a) \\
& =\sum_{a^{2}+b^{2} \leqslant R} e^{2 \pi i n g(a, b)} \sum_{k=1}^{a} f_{k} e^{2 \pi i b k / a} \\
& =\sum_{2 \leqslant a \leqslant \sqrt{R}} \sum_{k=1}^{a} f_{k} \sum_{b \leqslant \min \left(a, \sqrt{R-a^{2}}\right)} e^{2 \pi i(b k / a+n g(a, b))} .
\end{aligned}
$$

Следовательно, справедлива оценка

$$
U_{n} \ll \sum_{2 \leqslant a \leqslant \sqrt{R}} \sum_{k=1}^{a} \tau(a)(n, a)^{1 / 2} a^{-1 / 2}\left|V_{n}\right|,
$$

где

$$
V_{n}=V_{n}(a)=\sum_{1 \leqslant b \leqslant \min \left(a, \sqrt{R-a^{2}}\right)} e^{-2 \pi i(k b / a+n g(a, b))}
$$

Слагаемые, отвечающие значениям $a \leqslant R^{0,3}$, при тривиальной оценке суммы $V_{n}=V_{n}(a)$ вносят в общую оценку модуля суммы $S_{m}, m=1,2,3,4$, вклад, не превосходящий величины порядка $R^{3 / 4} \ln R$.

При выполнении неравенства $\left|k+n(a g(a, b))_{a}^{\prime}\right| \geqslant 1$ сумму $V_{n}$ оцениваем по первой производной, при выполнении противоположного неравенства $\left|k+n(a g(a, b))_{a}^{\prime}\right|<1$ сумму $V_{n}$ оцениваем по второй производной функщии, стоящей в экспоненте. Это дает нам для всех оставшихся значений $a$ оценку $S_{m}, m=1,2,3,4$, вида

$$
S_{m} \leqslant R^{3 / 4} \ln R
$$

Теорема доказана.

\section{Список литературы}

1. Архипов Г. И., Чубариков В. Н., О числе точек решетки в трехмерном пространстве Лобачевского. Вестник Московского ун-та. Сер. 1, Математика. Механика (1994) 2, 24-27.

2. Архипов Г. И., Чубариков В. Н., Оценка снизу первого собственного значения оператора Бельтрами-Лапласа в трехмерном пространстве. Труды семинара им. И. Г. Петровского (2002) 22, 22-36.

3. Архипов Г. И., Садовничий В. А., Чубариков В. Н., Лекции по математическому анализу. Дрофа, Москва, 2004.

4. Виноградов И. М., Элементы высшей математики. Высшая школа, Москва, 1999.

5. Виноградов И. М., Особые варианты метода тригонометрических сумм. Наука, Москва, 1976.

6. Груневальд Ф., Меннике Й., Эльстродт Ю., Группы, действующие на гиперболическом пространстве. МЦНМО, Москва, 2003.

7. Delsarte J., Sur le gitter fuchsien. C. R. Acad. Sci. Paris (1942) 214, 147-149. 
8. Delsarte J., Le gitter fuchsien. In: Oeuvres de Jean Delsarte II. Paris, 1971, pp. 829-845.

9. Graham S. W., Kolesnik G., Van der Corput's method of exponential sums. Cambridge Univ. Press, Cambridge, 1991.

10. Huber H., Über eine neue Klasse automorphe Funktionen und eine Gitterpunktproblem in der hyperbolischen Ebene. Comment. Math. Helv. (1956) 30, 20-62.

11. Huber H., Zur analytischen Theorie hyperbolischen Raumformen und Bewegungsgruppen. I. Math. Ann. (1959) 138, 1-26.

12. Huber H., Zur analytischen Theorie hyperbolischen Raumformen und Bewegungsgruppen. II. Math. Ann. (1961) 142, 385-398.

13. Huber H., Zur analytischen Theorie hyperbolischen Raumformen und Bewegungsgruppen. II. Math. Ann. (1961) 143, 463-464.

14. Huxley N. M., Area, lattice points, and exponential sums. Oxford Univ. Press, Oxford, 1996.

15. Lax P. D., Phillips R. S., The asymptotic distribution of lattice points in Euclidean and non-Euclidean spaces. J. Funct. Anal. (1982) 46, №3, 280-350.

16. Левитан Б. М., Асимптотические формулы для числа точек решетки в пространствах Евклида и Лобачевского. Успехи матем. наук (1987) 42, №3(255), 13-38.

17. Суги М., Целые точки в областях на плоскости Лобачевского. Труды Математического ин-та им. В. А. Стеклова РАН (1994) 207, 299-325.

18. Хооли К., Применения методов решета в теории чисел. Наука, Москва, 1987.

Статья поступила 22.11.2005. 